\title{
Cross-References to Entries by Exposure
}

Many readers will want to know which toxics they may be exposed to in various places or as the result of certain activities. Such information is provided in the following lists of entries from Part II, grouped by place or activity.

\section{AIR POLLUTANTS (INDOOR):} acrolein, asbestos, benzo[a]pyrene, carbon monoxide, chlordane, chloroform, dichlorvos, di(2-ethylhexyl)pthalate, ethylene dibromide and ethylene dichloride, extremely low frequency electromagnetic fields, formaldehyde, heptachlor, mineral fibers, napthalene, nitrogen dioxide, noise, particulate matter, radon, styrene, tetrachloroethylene, toluene, trichloroethylene, vinyl chloride, xylene.

2. AIR POLLUTANTS (OUTDOOR): acetic acid, arsenic, asbestos, benzene, benzo[a]pyrene, carbon black, carbon monoxide, CFCs, chloroform, chromium, creosote, dioxin, extremely low frequency electromagnetic fields, ethylene oxide, formaldehyde, lead, methylene chloride, nitrogen dioxide, noise, ozone, particulate matter, plutonium, radon, styrene, sulfur dioxide, tetrachloroethylene, toluene, ultraviolet light, vinyl chloride, vinylidene chloride.

3. AUTOMOBILE-RELATED ACTIVITIES: asbestos, asphalt, benzene, benzo[a]pyrene, BHT and BHA, cadmium, carbon monoxide, chloroform, ethylene dibromide and ethylene dichloride, formaldehyde, lead, nitrogen dioxide, noise, ozone, particulate matter, toluene, vinyl chloride.

4. FIRES (substances that result from the burning of insulation, plastics, fabrics, and other household products, as well as from controlled fires in the wood- stove or fireplace): benzo[a]pyrene, CFCs, cadmium, carbon monoxide, chloroform, creosote, dioxin, formaldehyde, nitrogen dioxide, particulate matter, PCBs.

\section{FOOD CONTAMINANTS AND} ADDITIVES: aflatoxin, alachlor, aldicarb, aldrin and dieldrin, aluminum arsenic, aspartame, azinophos-methyl, barium, benomyl, benzo[a]pyrene, beryllium, BHT and BHA, cadmium, caffeine, captan (and captafol, folpet), carbaryl, carrageenan, cesium-137, chlordane, chloroform, 2,4-D, daminozide, DDT, diazinon, di(2-ethylhexyl)pthalate, EDB and EDC, fluoride, food colors, fosetyl Al, glyphosate, heptachlor, iodine-131, lead, lindane, malathion, mancozeb (under EBCDs), mercury, MSG, nickel, nitrates (and nitrites, nitrosamines), parathion, PCBs, pyrethrum, saccharin, selenium, strontium90, sulfites, sulfur dioxide, 2,4,5-T, tetrachloroethylene, trichloroethylene, tritium, vinyl chloride, vinylidene chloride.

6. GARDEN AND LAWN ACTIVITIES: aldrin and dieldrin, arsenic, azinophos-methyl, benomyl, captan, carbaryl, DDT, diazinon, dioxin, glyphosate, lindane, malathion, noise, pyrethrum.

7. HOBBIES AND CRAFTS: acetic acid, acetone, benzene, cadmium, chromium, formaldehyde, dioxane, hydroquinone, methylene chloride, methyl ethyl ke- 
tone, noise, tetrachloroethylene, trichloroethylene, xylene.

8. HOUSEHOLD ACTIVITIES:

a. Bathroom (including cosmetics): acetic acid, acetone, ammonia, chlorine (and chloride, hydrochloric acid, hypochloric acid, and hypochlorite), fluoride, food colors, formaldehyde, hydroquinone, oxalic acid, xylene.

b. Kitchen (nonfood hazards listed here, but see also number 5): benzene, carbon monoxide, extremely low frequency electromagnetic fields, formaldehyde, microwave and radio frequency radiation, nitrogen dioxide, particulate matter, radiation, noise, tetrachloroethylene.

c. Miscellaneous Housebold Products: acetone, ammonia, barium, benzene, carbon black, dichlorvos, dioxane, di(2-ethylhexyl)phthalate, extremely low frequency electromagnetic fields, formaldehyde, laser light, methylene chloride, methyl ethyl ketone, mineral fibers, naphthalene, nickel, noise, oxalic acid, sodium hydroxide, trichloroethylene, warfarin, xylene.

9. WATER POLLUTANTS: aldicarb, aldrin and diedrin, aluminum, arsenic, barium, benzene, benzo[a]pyrene, beryllium, cadmium, captafol, chlordane, chromium, 2,4-D, DDT, diazinon, dioxin, EBDCs, fluoride, heptachlor, lead, lindane, malathion, nitrates (and nitrites and nitrosamines), parathion, selenium, 2,4,5-T, toluene, tritium.

10. SOIL CONTAMINANTS: acrolein, aldrin and dieldrin, arsenic, benzo[a]pyrene, bis(chloromethyl)ether, cadmium, cesium-137, chlordane, creosote, 2,4-D, DDT, dioxin, heptachlor, lead, lindane, $\mathrm{PCB}$ and $\mathrm{PBB}$, plutonium, strontium90, 2,4,5-T.

11. TOBACCO SMOKE: acrolein, arsenic, benzo[a]pyrene, cadmium, car- bon monoxide, ethylene oxide, formaldehyde, nickel, nicotine, nitrates (and nitrites and nitrosamines), particulate matter, radon, toluene, vinyl chloride.

12. ACCIDENTAL SPILLS (for example, truck or train accidents and pipeline ruptures): ammonia, benzene, chlorine (and hydrogen chloride, hydrochloric acid, and hypochlorite).

13. WORKPLACE CONTAMINANTS:

a. Office: asbestos, benzene, extremely low frequency electromagnetic fields, formaldehyde, laser light, microwave and radio frequency fields, noise, ozone, PCBs, trichloroethylene.

b. Agriculture, borticulture, and forestry: alachlor, aldicarb, aldrin and dieldrin, ammonia, azinophosmethyl, benomyl, BHT and BHA, captan (and captafol and folpet), carbon tetrachloride, 2,4-D, daminozide, DDT, dicamba, EBCDs, glyphosate, heptachlor, lindane, malathion, nitrates (and nitrites and nitrosamines), noise, paraquat, parathion, sulfur dioxide, 2,4,5-T.

c. Services (including schools, hospitals and doctors' offices, laboratories, gas stations, and dry cleaners; see also number $8 \mathrm{~b}$ ): acetone, asbestos, barium, benzene, bis(chloromethyl)ether, CFCs, di(2-ethylhexyl)pthalate, ethylene dibromide (and ethylene dichloride), ethylene oxide, iodine-131, laser light, mercury, microwave and radio frequency radiation, oxalic acid, tetrachloroethylene, trichloroethylene, tritium, ultrasound, ultraviolet radiation.

d. Nuclear Industry: beryllium, cesium137 , iodine-131, plutonium, radon, strontium-90, tritium.

e. Fossil Fuel Mining and Combustion (including electric power plants and incinerators): acetic acid, acetone, aluminum, arsenic, benzene, benzo[a]pyrene, beryllium, cadmium, chromium, extremely low frequency 
electromagnetic fields, lead, nitrogen dioxide, noise, particulate matter, sulfur dioxide.

f. Chemical Industries (including pesticides, plastics, rubber, cosmetics, and solvents): acetic acid, acetone, acrolein, aldicarb, aldrin and dieldrin, ammonia, arsenic, azinophosmethyl, benomyl, benzene, BHT and $\mathrm{BHA}$, bis(chloromethyl)ether, carbon black, carbon tetrachloride, CFCs, chlordane, chlorine (and hydrogen chloride, hydrochloric acid, and hypochlorite), chloroform, chromium, creosote, dioxane, dioxin, di(2-ethylhexyl)phthalate, ethylene oxide, lindane, malathion, methyl ethyl ketone, methylene chloride, mercury, naphthalene, parathion, sodium hydroxide, styrene, tetrachloroethylene, toluene, trichloroethylene, vinyl chloride, vinylidene chloride, xylene.

g. Metals Industries: acetone, aluminum, arsenic, beryllium, carbon tetrachloride, cadmium, chromium, dioxane, lead, mercury, methyl ethyl ketone, nickel, noise, oxalic acid, particulate matter, saccharin, sulfur dioxide, tetrachloroethylene, trichloroethylene.

h. Electronics Industries: beryllium, cadmium, carbon tetrachloride, CFCs, lead, mercury, selenium, tetrachloroethylene.

i. Paper and Wood Products Industries: acetic acid, arsenic, chlorine (and hydrogen chloride, hydrochloric acid, and hypochlorite), chloroform, chromium, creosote, dioxin, formaldehyde, noise, sodium hydroxide. j. Textiles Industries: acetic acid, cadmium, chromium, benzene, bis(chloromethyl)ether, chloroform, formaldehyde, tetrachloroethylene.

14. RADIOACTIVE SUBSTANCES: cesium-137, iodine-131, plutonium, radon, strontium-90, tritium.

15. METALS (or metal-like elements): aluminum, arsenic, barium, beryllium, cadmium, chromium, lead, mercury, nickel, selenium.

16. SOLVENTS: acetic acid, acetone, acrolein, ammonia, benzene, bis(chloromethyl)ether, CFCs, carbon tetrachloride, chloroform, dioxane, ethylene glycol, formaldehyde, methylene chloride, methyl ethyl ketone, oxalic acid, tetrachloroethylene, toluene, trichloroethylene, vinyl chloride, xylene.

17. PESTICIDES: alachlor, aldicarb, aldrin and dieldrin, azinophos-methyl, benomyl, captan (and captafol and folpet), carbaryl, chlordane, creosote, 2,4-D, daminozide, diazinon, dicamba, DDT, dichlorvos, EBDCs, ethylene dibromide and ethylene dichloride, fosetyl $\mathrm{Al}$, glyphosate, heptachlor, lindane, malathion, paraquat, parathion, pyrethroids, 2,4,5-T, warfarin.

18. TOXICS OF PARTICULAR ENVIRONMENTAL CONCERN : aldicarb, aldrin and dieldrin, carbaryl, carbon dioxide, CFCs, 2,4-D, DDT, EBDCs, heptachlor, lindane, nitrogen dioxide, ozone, parathion, $\mathrm{PCBs}$, selenium, sulfur dioxide, $2,4,5-\mathrm{T}$, ultraviolet radiation. 